\title{
New results for an open time-dependent scheduling problem
}

\author{
Stanisław Gawiejnowicz ${ }^{1}\left[\right.$ : Wiesław Kurc ${ }^{1}$
}

Published online: 13 July 2020

(c) The Author(s) 2020

\begin{abstract}
We present several new results for a single machine time-dependent scheduling problem of minimizing the total completion time of a set of linearly deteriorating jobs with unit basic processing times. First, we show new properties of cyclic transformations of V-shaped sequences for this problem. Next, applying the results, we prove a new necessary condition of schedule optimality for the considered problem, which decreases the previous bound on the cardinality of the set containing all possible optimal schedules by a multiplicative factor which is at most proportional to the reciprocal of the square root of the number of jobs. Finally, we compare the strength of the new and the previous necessary conditions by estimation of the numbers of schedules satisfying the respective conditions.
\end{abstract}

Keywords Scheduling $\cdot$ Single machine $\cdot$ Deteriorating jobs $\cdot$ Total completion time $\cdot$ V-shapeness $\cdot$ Necessary conditions of optimality

\section{Introduction}

We consider the following scheduling problem with variable job processing times. Jobs $J_{0}, J_{1}, \ldots, J_{n}$, where $n>2$, have to be scheduled on a single machine, starting from time 0 . All the jobs are independent, i.e. there are no precedence constraints among the jobs. The processing time of job $J_{j}$ linearly deteriorates in time and is equal to $p_{j}(t)=1+\alpha_{j} t$, where $t$ is the starting time of the job, $\alpha_{j}>0$ is its deterioration rate and $0 \leq j \leq n$. Let $a^{\circ}=\left(a_{0}, a_{1}, \ldots, a_{n}\right)$ denote the initial sequence of coefficients $a_{j}=1+\alpha_{j}>1$, where $0 \leq j \leq n$. Let $\mathcal{P}\left(a^{\circ}\right)$ denote the set of all permutations of sequence $a^{\circ}$. Notice that any sequence $a^{\pi} \in \mathcal{P}\left(a^{\circ}\right)$, in which the elements of sequence $a^{\circ}$ are ordered according to a permutation $\pi=\left(\pi_{0}, \pi_{1}, \ldots, \pi_{n}\right)$ of indices $0,1, \ldots, n$, corresponds to a schedule in which the job with index $\pi_{0}$ starts at time 0 and all remaining jobs are scheduled one by one without idle times. Therefore, in the paper we use terms 'sequence' and 'schedule' interchangeably, equating a given sequence $a^{\pi} \in \mathcal{P}\left(a^{\circ}\right)$ with the schedule corresponding to $a^{\pi}$. Hence, for a given $a^{\pi}=\left(a_{\pi_{0}}, a_{\pi_{1}}, \ldots, a_{\pi_{n}}\right) \in \mathcal{P}\left(a^{\circ}\right)$, where $a_{\pi_{j}}=1+\alpha_{\pi_{j}}$ for $0 \leq j \leq n$, the completion time of

Stanisław Gawiejnowicz

stgawiej@amu.edu.pl

1 Faculty of Mathematics and Computer Science, Adam Mickiewicz University Poznań, Uniwersytetu Poznańskiego 4, 61-614 Poznań, Poland the $j$ th job in the schedule corresponding to sequence $a^{\pi}$ is equal to

$$
\begin{aligned}
C_{j}\left(a^{\pi}\right)= & C_{j-1}\left(a^{\pi}\right)+p_{\pi_{j}}\left(C_{j-1}\left(a^{\pi}\right)\right)=1 \\
& +a_{\pi_{j}} C_{j-1}\left(a^{\pi}\right),
\end{aligned}
$$

with $C_{0}\left(a^{\pi}\right)=0+1+\alpha_{\pi_{0}} \cdot 0=1$, since job $J_{\pi_{0}}$ starts at time $t=0$. Then, the problem under consideration is to find a sequence corresponding to a schedule with minimal total completion time, i.e. to find $a^{\sigma} \in \mathcal{P}\left(a^{\circ}\right)$ such that

$$
\sum_{j=0}^{n} C_{j}\left(a^{\sigma}\right)=\min _{a^{\pi} \in \mathcal{P}\left(a^{\circ}\right)}\left\{\sum_{j=0}^{n} C_{j}\left(a^{\pi}\right)\right\},
$$

where job completion times $C_{j}\left(a^{\pi}\right)$ are defined by the recurrence equation (1). Since sequences in which two equal elements have been mutually exchanged are identical, and since the order of jobs with the same deterioration rate has no impact on the value of the $\sum C_{j}$ criterion, we assume that $a_{i} \neq a_{j}$ whenever $i \neq j$ and $0 \leq i, j \leq n$. The problem formulated as above, in three-field notation denoted as $1\left|p_{j}(t)=1+\alpha_{j} t\right| \sum C_{j}$, in this paper is called problem $(P)$.

Problem $(P)$ is a time-dependent scheduling problem, where deteriorating job processing times are non-decreasing functions of the starting times of the respective jobs. Scheduling problems of this kind are dynamic in the sense that job 
processing times are not fixed but change in time. Research on time-dependent scheduling problems was started about 40 years ago, quickly evolving into a self-contained research domain in scheduling theory. We refer the reader to reviews by Cheng et al. (2004) and Gawiejnowicz (2020a), and books by Gawiejnowicz (2008), Agnetis et al. (2014), Strusevich and Rustogi (2017) and Gawiejnowicz (2020b) for more details on the subject.

In this paper, we prove several new results for problem $(P)$ and show some their consequences. The paper is organized as follows. In Sect. 2, we summarize the previous research on problem $(P)$. In Sect. 3, we formulate and prove some preliminary results which concern cyclic shifts of V-shaped sequences. In Sect. 4, we introduce a new necessary condition of schedule optimality for problem $(P)$ which lowers the previous bound on the cardinality of the set containing all possible optimal schedules for $(P)$ by a multiplicative factor at most proportional to the reciprocal of the square root of the number of jobs. In Sect. 5, we compare the power of the new and the previous necessary condition of schedule optimality for problem $(P)$, estimating the numbers of schedules which satisfy the respective condition. Finally, in Sect. 6, we present several numerical examples which illustrate the results of Sect. 5. We complete the paper with Sect. 7, including conclusions and remarks on future research.

\section{Previous research on problem $(P)$}

Research on problem $(P)$ has more than a 25-year-old history that was initiated by Mosheiov (1991), who formulated the problem and proved some its properties.

The first property specifies which job is the first one in an optimal schedule.

Property 1 (Mosheiov 1991) In an optimal schedule for problem $(P)$ the first scheduled job is the one with the maximal deterioration rate.

In view of Property 1 , in sequence $a^{\pi} \in \mathcal{P}\left(a^{\circ}\right)$ corresponding to an optimal schedule the element $a_{\pi_{0}}$ corresponds to the greatest deterioration rate, $a_{\pi_{0}}=\max \left\{1+\alpha_{j}: 0 \leq\right.$ $j \leq n\}$.

The second property shows a symmetry of optimal schedules for problem $(P)$.

Property 2 (Mosheiov 1991) If $\left(a_{\pi_{0}}, a_{\pi_{1}}, \ldots, a_{\pi_{n}}\right) \in \mathcal{P}\left(a^{\circ}\right)$ is an optimal schedule for problem $(P)$, then schedule $\left(a_{\pi_{0}}, a_{\pi_{n}}, \ldots, a_{\pi_{1}}\right) \in \mathcal{P}\left(a^{\circ}\right)$ is optimal for $(P)$ as well.

By Property 2, for any instance of problem $(P)$ there exist at least two optimal schedules that are symmetric to each other, starting from the second position.

Before we formulate the third property, we recall a definition.
Definition 1 A sequence $\left(a_{\pi_{0}}, a_{\pi_{1}}, \ldots, a_{\pi_{n}}\right)$ is said to be $V$ shaped, if there exists $0 \leq m \leq n$ such that $a_{\pi_{k}} \geq a_{\pi_{k+1}}$ for any $0 \leq k \leq m-1$ and $a_{\pi_{k}} \leq a_{\pi_{k+1}}$ for any $m \leq k \leq n-1$.

From Definition 1, it follows that a sequence is V-shaped if elements before and after the minimal element in the sequence are in the non-increasing and non-decreasing order, respectively.

The third property concerns the shape of an optimal schedule for the considered problem. We call the property the first necessary condition of schedule optimality for problem $(P)$.

Property 3 (Mosheiov 1991) If $\left(a_{\pi_{0}}, a_{\pi_{1}}, \ldots, a_{\pi_{n}}\right) \in \mathcal{P}\left(a^{\circ}\right)$ is an optimal schedule for problem $(P)$, then it is $V$-shaped.

By Property 3, jobs in an optimal schedule are scheduled in the non-increasing or non-decreasing order with respect to job deterioration rates if the jobs are placed before or after the job with the smallest deterioration rate, respectively.

Mosheiov (1994) considered a similar to $(P)$ single machine scheduling problem with proportionally deteriorating job processing times, i.e. $p_{j}(t)=\alpha_{j} t$, where $\alpha_{j}>0$ for $1 \leq j \leq n$ and $t>0$, and showed that it is solvable in $O(n \log n)$ time by scheduling jobs in the non-decreasing order of job deterioration rates. Gawiejnowicz et al. (2006a) defined functions of job deterioration rates, called signatures, proved the basic properties of these functions and proposed two $O(n \log n)$ signature-based greedy algorithms for problem $(P)$. Gawiejnowicz et al. (2006b) proved several properties of two generalizations of problem $(P)$, in which the aim is to minimize the weighted sum of the $C_{\max }$ and $\sum C_{j}$ criteria or to find a Pareto optimal schedule for them. Kubale and Ocetkiewicz (2009) proposed an $O(n \log n)$ algorithm for $(P)$, provided that inequality $\alpha_{i}>\alpha_{j}$ implies inequality $\alpha_{i} \geq \frac{\alpha_{\min }+1}{\alpha_{\min }} \alpha_{j}+\frac{1}{\alpha_{\min }}$ for any $i \neq j$, where $\alpha_{\min }$ is the smallest deterioration rate. For a special case of $(P)$ in which all deterioration rates are not smaller than a certain constant $u>0$, Ocetkiewicz (2010) proposed a fully polynomial-time approximation scheme (FPTAS) running in $O\left(n^{1+6 \log _{1+u} 2}\left(\frac{1}{\epsilon}\right)^{2 \log _{1+u} 2}\right)$ time, where $\epsilon>0$ is a given accuracy. For general case of problem $(P)$, Ocetkiewicz (2013) proposed a branch-and-bound algorithm. Gawiejnowicz and Kurc (2015) shown that counterparts of Property 3 also hold for time-dependent scheduling problems with more general criterion functions than the total completion time, considering a generalization of problem $(P)$ with the objective to minimize the $l_{p}$ norm $\left\|C\left(a^{\pi}\right)\right\|_{p}=\left(\sum_{j=0}^{n} C_{j}\left(a^{\pi}\right)^{p}\right)^{\frac{1}{p}}$ of vector $C\left(a^{\pi}\right)=$ $\left(C_{0}\left(a^{\pi}\right), C_{1}\left(a^{\pi}, \ldots, C_{n}\left(a^{\pi}\right)\right)\right.$, where $1 \leq p \leq+\infty$. The authors showed that the symmetry of optimal schedules that holds for the total completion time, i.e. for $p=1$, may hold for the $l_{p}$ norm only for some $p>1$, while the $\mathrm{V}$-shapeness of optimal schedules for the $l_{p}$ norm and bounded logarithmic 
growth of the criterion function values hold both for $p=1$ and for all $p>1$.

Despite so long research history, the time complexity of problem $(P)$ is still unknown. We only know that Properties 1-3 allow us to decrease the cardinality of the set containing all possible optimal schedules for $(P)$ from $O(n !)$ to $O\left(2^{n-1}\right)$. Hence, finding a new property for $(P)$ is a challenging research task. In this paper, we take a step in this direction, proving a new necessary condition for $(P)$, which implies the decrease in the bound $O\left(2^{n-1}\right)$ by the factor between $1-\frac{1}{\sqrt{n}} \times 2^{O(1)}$ and $O\left(n^{-\frac{1}{2}}\right)$ for sequences $a^{\circ}$ in which their minimal and maximal elements are sufficiently close, and by the factor $1-\frac{2^{-n}}{\sqrt{n}} \times 2^{O(1)}$ for arbitrary sequences $a^{\circ}$.

\section{Preliminary results}

Since the results presented further on concern arbitrary but fixed sequences from the set $\mathcal{P}\left(a^{\circ}\right)$, starting from this section we use simplified notation in which we omit superscripts related to permutations, e.g. we write $a$ instead of $a^{\pi}$. Similarly, we denote the full form of considered sequences, taking into account the properties of problem $(P)$. For example, we write $a=\left(a_{1}, \ldots, a_{n}\right) \in \mathcal{P}\left(a^{\circ}\right)$ instead of $a^{\pi}=\left(a_{\pi_{0}}, a_{\pi_{1}}, \ldots, a_{\pi_{n}}\right) \in \mathcal{P}\left(a^{\circ}\right)$, omitting $a_{\pi_{0}}$, since it is fixed by Property 1 .

Throughout the paper, we assume that any V-shaped sequence is composed of the left and the right branch. If one of the branches is empty, the V-shaped sequence becomes a non-increasing or non-decreasing sequence. A $\mathrm{V}$-shaped sequence with non-empty branches may be composed of distinct or non-distinct elements. Since any pair of non-distinct elements of a sequence decreases the number of V-shaped sequences which we can generate from this sequence, we assume that $\mathrm{V}$-shaped sequences are composed only of distinct elements. We also distinguish the case when the minimal element of a V-shaped sequence belongs either to the left or to the right branch of the sequence.

\subsection{Perturbation formulae}

Let $a\left(a_{r} \leftrightarrow a_{q}\right)$ denote sequence $a=\left(a_{1}, a_{2}, \ldots, a_{n}\right)$ with elements $a_{r}$ and $a_{q}$ mutually exchanged, where $1 \leq r<$ $q \leq n$. Observe that for given $a$ and $b=a\left(a_{r} \leftrightarrow a_{q}\right)$, where $r$ and $q$ are indices defined above, and for $j=0,1, \ldots, n$ there holds the equality (Gawiejnowicz et al. 2006b):

$$
\begin{aligned}
& C_{j}(b)-C_{j}(a) \\
& = \begin{cases}\left(\frac{a_{r}-a_{q}}{a_{q}}\right) \sum_{i=r}^{q-1} \prod_{k=i+1}^{j} a_{k}, & 1 \leq r<q \leq j \leq n, \\
\left(\frac{a_{q}-a_{r}}{a_{r}}\right) \sum_{i=0}^{r-1} \prod_{k=i+1}^{j} a_{k}, & 1 \leq r \leq j<q \leq n, \\
0, & 0 \leq j<r<q \leq n .\end{cases}
\end{aligned}
$$

Summing formula (2) side by side for $j=0,1, \ldots, n$, we have

$$
\begin{aligned}
& \|C(b)\|_{1}-\|C(a)\|_{1} \\
& =\left(a_{q}-a_{r}\right)\left(\sum_{j=r}^{q-1} \frac{1}{a_{r}} \sum_{i=0}^{r-1}\left(a_{i+1} \cdots a_{r} \cdots a_{j}\right)\right. \\
& \left.\quad-\sum_{j=q}^{n} \frac{1}{a_{q}} \sum_{i=r}^{q-1}\left(a_{i+1} \cdots a_{q} \cdots a_{j}\right)\right) .
\end{aligned}
$$

By simplifying the second difference in Eq. (3), we obtain equality

$$
\begin{aligned}
\|C(b)\|_{1}-\|C(a)\|_{1} \\
=\left(a_{q}-a_{r}\right)\left[\left(\sum_{j=r}^{q-1} \prod_{k=r+1}^{j} a_{k}\right)\left(\sum_{i=0}^{r-1} \prod_{k=i+1}^{r-1} a_{k}\right)\right. \\
\left.-\left(\sum_{i=r}^{q-1} \prod_{k=i+1}^{q-1} a_{k}\right)\left(\sum_{j=q}^{n} \prod_{k=q+1}^{j} a_{k}\right)\right]
\end{aligned}
$$

Mutually exchanging in Eq. (4) two consecutive elements with indices $r$ and $q=r+1$, where $1 \leq r \leq n-1$, we obtain equality

$$
\begin{aligned}
& \|C(b)\|_{1}-\|C(a)\|_{1} \\
& =\left(a_{r+1}-a_{r}\right)\left(\sum_{j=0}^{r-1} \prod_{k=j+1}^{r-1} a_{k}-\sum_{i=r+1}^{n} \prod_{k=r+2}^{i} a_{k}\right) .
\end{aligned}
$$

\subsection{Cyclic shifts}

Given sequence $a=\left(a_{1}, \ldots, a_{r}, \ldots, a_{q}, \ldots, a_{n}\right)$, where $1 \leq r<q \leq n$, let $a\left(a_{r} \leftarrow a_{q}\right)$ denote sequence $a$ in which element $a_{q}$ has been moved immediately before element $a_{r}$. Similarly, let $a\left(a_{r} \rightarrow a_{q}\right)$ denote sequence $a$ in which element $a_{r}$ has been moved immediately after element $a_{q}$, i.e. let

$a\left(a_{r} \leftarrow a_{q}\right)=\left(a_{1}, \ldots, a_{r-1}, a_{q}, a_{r}, \ldots, a_{q-1}, a_{q+1}, \ldots, a_{n}\right)$ 
and

$a\left(a_{r} \rightarrow a_{q}\right)=\left(a_{1}, \ldots, a_{r-1}, a_{r+1}, \ldots, a_{q}, a_{r}, a_{q+1}, \ldots, a_{n}\right)$.

We call $a\left(a_{r} \leftarrow a_{q}\right)$ and $a\left(a_{r} \rightarrow a_{q}\right)$ the cyclic shifts of sequence $a$.

Notice that given $a$ and $1 \leq r<q \leq n$, we can obtain the cyclic shifts $a\left(a_{r} \leftarrow a_{q}\right)$ and $a\left(a_{r} \rightarrow a_{q}\right)$ by consecutive transpositions of suitable pairs of elements in $a$. Namely, if we define $\bar{a}^{0}=a$, then

$$
\begin{aligned}
\bar{a}^{1}= & \bar{a}^{0}\left(\bar{a}_{q}^{0} \leftrightarrow \bar{a}_{q-1}^{0}\right), \\
\bar{a}^{2}= & \bar{a}^{1}\left(\bar{a}_{q-1}^{1} \leftrightarrow \bar{a}_{q-2}^{1}\right), \\
\cdots & \quad \cdots, \\
\bar{a}^{q-r}= & \bar{a}^{q-r-1}\left(\bar{a}_{r}^{q-r-1} \leftrightarrow \bar{a}_{r+1}^{q-r-1}\right)
\end{aligned}
$$

or, in explicit form,

$$
\begin{array}{ll}
\bar{a}^{0} & =\left(a_{1}, \ldots, a_{r}, a_{r+1}, a_{r+2}, \ldots, a_{q-1}, a_{q}, a_{q+1}, \ldots, a_{n}\right), \\
\bar{a}^{1} & =\left(a_{1}, \ldots, a_{r}, a_{r+1}, a_{r+2}, \ldots, a_{q}, a_{q-1}, a_{q+1}, \ldots, a_{n}\right), \\
\ldots & \ldots \ldots, \\
\bar{a}^{q-r-1} & =\left(a_{1}, \ldots, a_{r}, a_{q}, a_{r+1}, \ldots, a_{q-1}, a_{q+1}, \ldots, a_{n}\right), \\
\bar{a}^{q-r} & =\left(a_{1}, \ldots, a_{q}, a_{r}, a_{r+1}, \ldots, a_{q-1}, a_{q+1}, \ldots, a_{n}\right),
\end{array}
$$

where box indicates the element $a_{q}$ which is shifted in sequence $a$. In other words, $\bar{a}^{1}$ is the result of transposition of the $(q-1)$ th and $q$ th components of sequence $\bar{a}^{0}=a, \bar{a}^{2}$ is the result of transposition of the $(q-2)$ th and $(q-1)$ th components of $\bar{a}^{0}=a$, etc. Finally, $\bar{a}^{q-r}$ is the result of transposition of the $r$ th and $(r+1)$ th components of $\bar{a}^{q-r-1}$. Therefore, we can identify $a\left(a_{r} \leftarrow a_{q}\right)$ with $\bar{a}^{q-r}$.

Similarly, defining $\underline{a}^{0}=a$, applying in $\underline{a}^{0}$ the transposition of elements $\underline{a}_{r}^{0}$ and $\underline{a}_{r+1}^{0}$ from the left to right and proceeding further as previously, we have

$$
\begin{aligned}
\underline{a}^{1}= & \underline{a}^{0}\left(\underline{a}_{r+1}^{0} \leftrightarrow \underline{a}_{r}^{0}\right), \\
\underline{a}^{2}= & \underline{a}^{1}\left(\underline{a}_{r+2}^{1} \leftrightarrow \underline{a}_{r+1}^{1}\right), \\
\cdots & \cdots, \\
\underline{a}^{q-r}= & \underline{a}^{q-r-1}\left(\underline{a}_{q}^{q-r-1} \leftrightarrow \underline{a}_{q-1}^{q-r-1}\right)
\end{aligned}
$$

or, in explicit form,

$$
\begin{array}{ll}
\underline{a}^{0} & =\left(a_{1}, \ldots, a_{r}, a_{r+1}, a_{r+2}, \ldots, a_{q-1}, a_{q}, a_{q+1}, \ldots, a_{n}\right), \\
\underline{a}^{1} & =\left(a_{1}, \ldots, a_{r+1}, a_{r}, a_{r+2}, \ldots, a_{q-1}, a_{q}, a_{q+1}, \ldots, a_{n}\right), \\
\ldots & \ldots \ldots, \\
\underline{a}^{q-r-1} & =\left(a_{1}, \ldots, a_{r+1}, a_{r+2}, a_{r+3}, \ldots, a_{r}, a_{q}, a_{q+1}, \ldots, a_{n}\right), \\
\underline{a}^{q-r} & =\left(a_{1}, \ldots, a_{r+1}, a_{r+2}, a_{r+3}, \ldots, a_{q}, a_{r}, a_{q+1}, \ldots, a_{n}\right) .
\end{array}
$$

Therefore, $a\left(a_{r} \rightarrow a_{q}\right)$ can be identified with $\underline{a}^{q-r}$.

Hence, in view of formulae (8)-(11), we use symbols $a\left(a_{r} \leftarrow a_{q}\right)$ and $a\left(a_{r} \rightarrow a_{q}\right)$ interchangeably with symbols $\bar{a}^{q-r}$ and $\underline{a}^{q-r}$, respectively.

Since below we consider V-shaped sequences in which the positions of some elements are distinguished, now we introduce two new terms.

Definition 2 Let $a=\left(a_{1}, \ldots, a_{m}, \ldots, a_{n}\right) \in \mathcal{P}\left(a^{\circ}\right)$ be a Vshaped sequence such that $a_{m}$ is the minimal element in $a$ and $1<m<n$. Index $r$ is said to be $m$-conjugated to index $q$, if $m<q \leq n, 1 \leq r<m$ and $a\left(a_{r} \leftarrow a_{q}\right)$ is V-shaped. Similarly, index $q$ is said to be $m$-conjugated to index $r$, if $1 \leq r<m, m<q \leq n$ and $a\left(a_{r} \rightarrow a_{q}\right)$ is V-shaped.

From Definition 2, it follows that cyclic shifts of a Vshaped sequence $a$ lead to sequences $a\left(a_{r} \leftarrow a_{q}\right)$ and $a\left(a_{r} \rightarrow a_{q}\right)$ which are V-shaped as well, whenever $r$ and $q$ are $m$-conjugated to each other. Notice also that indices $r$ and $q$ need not be mutually $m$-conjugated.

The behaviour of criterion $\|\cdot\|_{1}$ for such cyclic shifts can be determined by means of some formulae. Before we present the formulae, we introduce two functions of sequence $a$.

Definition 3 Let

$$
\begin{aligned}
\Delta_{k, q}(a)= & \sum_{i=1}^{q-k-1} \prod_{j=i}^{q-k-1} a_{j}-\sum_{i=q-k+1}^{q-1} \prod_{j=q-k+1}^{i} a_{j} \\
& -\frac{1}{a_{q}} \sum_{i=q+1}^{n} \prod_{j=q-k+1}^{i} a_{j}
\end{aligned}
$$

and

$$
\begin{aligned}
\nabla_{k, r}(a)= & \frac{1}{a_{r}} \sum_{i=1}^{r-1} \prod_{j=i}^{r+k-1} a_{j}+\sum_{i=r+1}^{r+k-1} \prod_{j=i}^{r+k-1} a_{j} \\
& -\sum_{i=r+k+1}^{n} \prod_{j=r+k+1}^{i} a_{j},
\end{aligned}
$$

where $1 \leq r<q \leq n$ and $k=1,2, \ldots, q-r$.

The next result shows that for fixed indices $r$ and $q$ the sequences of functions $\Delta_{k, q}(a)$ and $\nabla_{k, r}(a)$ are monotonic. We omit the proof of the result, since it follows directly from Definition 3.

Lemma 1 Let $r$ and $q$ be fixed, $1 \leq r<q \leq n$. Then, there hold inequalities

$\Delta_{1, q}(a) \geq \Delta_{2, q}(a) \geq \cdots \geq \Delta_{q-r, q}(a)$

and

$\nabla_{1, r}(a) \leq \nabla_{2, r}(a) \leq \cdots \leq \nabla_{q-r, r}(a)$. 
The last result in this section concerns differences $\left\|C\left(\bar{a}^{q-r}\right)\right\|_{1}-\|C(a)\|_{1}$ and $\left\|C\left(\underline{a}^{q-r}\right)\right\|_{1}-\|C(a)\|_{1}$.

Lemma 2 If $a=\left(a_{1}, \ldots, a_{r}, \ldots, a_{q}, \ldots, a_{n}\right)$ with $1 \leq r<$ $q \leq n$, then

$$
\left\|C\left(\bar{a}^{q-r}\right)\right\|_{1}-\|C(a)\|_{1}=\sum_{k=1}^{q-r}\left(a_{q}-a_{q-k}\right) \Delta_{k, q}(a)
$$

and

$$
\left\|C\left(\underline{a}^{q-r}\right)\right\|_{1}-\|C(a)\|_{1}=\sum_{k=1}^{q-r}\left(a_{r+k}-a_{r}\right) \nabla_{k, r}(a) .
$$

Proof To prove formulae (12) and (13), we apply consecutive pairwise transpositions. Let $1 \leq r<q \leq n$.

In order to prove (12), we proceed as follows. Let

$$
\begin{aligned}
\bar{a}^{s}= & \left(\bar{a}_{1}^{s}, \ldots, \bar{a}_{r-1}^{s}, \bar{a}_{r}^{s}, \bar{a}_{r+1}^{s}, \bar{a}_{r+2}^{s},\right. \\
& \left.\ldots, \bar{a}_{q-2}^{s}, \bar{a}_{q-1}^{s}, \bar{a}_{q}^{s}, \bar{a}_{q+1}^{s}, \ldots, \bar{a}_{n}^{s}\right)
\end{aligned}
$$

for $s=0,1,2, \ldots, q-r$. First, applying Eq. (5), we calculate for $s=1,2, \ldots, q-r$ differences $\delta_{s}-\delta_{s-1}$, where $\delta_{s}=\left\|C\left(\bar{a}^{s}\right)\right\|_{1}$. Then,

$$
\begin{aligned}
& \delta_{1}-\delta_{0}=\left(\bar{a}_{q}^{0}-\bar{a}_{q-1}^{0}\right)\left(\sum_{j=0}^{q-2} \prod_{k=j+1}^{q-2} \bar{a}_{k}^{0}-\sum_{i=q}^{n} \prod_{k=q+1}^{i} \bar{a}_{k}^{0}\right), \\
& \delta_{2}-\delta_{1}=\left(\bar{a}_{q-1}^{1}-\bar{a}_{q-2}^{1}\right)\left(\sum_{j=0}^{q-3} \prod_{k=j+1}^{q-3} \bar{a}_{k}^{1}-\sum_{i=q-1}^{n} \prod_{k=q}^{i} \bar{a}_{k}^{1}\right), \\
& \ldots \ldots \ldots, \\
& \delta_{q-r}-\delta_{q-r-1}=\left(\bar{a}_{r+1}^{q-r-1}-\bar{a}_{r}^{q-r-1}\right)\left(\sum_{j=0}^{r-1} \prod_{k=j+1}^{r-1} \bar{a}_{k}^{q-r-1}\right. \\
& \left.-\sum_{i=r+1}^{n} \prod_{k=r+2}^{i} \bar{a}_{k}^{q-r-1}\right) .
\end{aligned}
$$

Next, we simplify the right sides of these equalities. Simplifying the right side of the equality for difference $\delta_{1}-\delta_{0}$, by (9) we have $\bar{a}_{k}^{0}=a_{k}$ for $k=1,2, \cdots, n$. Hence,

$\bar{a}_{q}^{0}-\bar{a}_{q-1}^{0}=a_{q}-a_{q-1}$

and, by Definition 3,

$\sum_{j=0}^{q-2} \prod_{k=j+1}^{q-2} \bar{a}_{k}^{0}-\sum_{i=q}^{n} \prod_{k=q+1}^{i} \bar{a}_{k}^{0}=\Delta_{1, q}\left(\bar{a}^{0}\right)$.

Similarly, simplifying the right side of the equality for difference $\delta_{2}-\delta_{1}$, by (9) we have $\bar{a}_{q-1}^{1}=a_{q}, \bar{a}_{q-2}^{1}=a_{q-2}$ and $\bar{a}_{k}^{1}=a_{k}$ for $k=1,2, \cdots, q-2, q+1, \cdots, n$. Hence,

$\bar{a}_{q-1}^{1}-\bar{a}_{q-2}^{1}=a_{q}-a_{q-2}$ and, by Definition 3,

$\sum_{j=0}^{q-3} \prod_{k=j+1}^{q-3} \bar{a}_{k}^{1}-\sum_{i=q-1}^{n} \prod_{k=q}^{i} \bar{a}_{k}^{1}=\Delta_{2, q}\left(\bar{a}^{1}\right)$.

In a similar way, we proceed for $s=3,4, \ldots, q-r-1$. Finally, simplifying the right side of the equality for difference $\delta_{q-r}-\delta_{q-r-1}$, we have, by (9), $\bar{a}_{r+1}^{q-r-1}=a_{q}$ and $\bar{a}_{r}^{q-r-1}=a_{r}$, whereas $\bar{a}_{k}^{q-r-1}=a_{k}$ for $k=1,2, \cdots, r$, $\bar{a}_{k}^{q-r-1}=a_{k-1}$ for $k=r+2, \cdots, q-1$ and $\bar{a}_{k}^{q-r-1}=a_{k}$ for $k=q+1, \cdots, n$. Hence,

$\bar{a}_{r+1}^{q-r-1}-\bar{a}_{r}^{q-r-1}=a_{q}-a_{r}$

and, by Definition 3,

$\sum_{j=0}^{r-1} \prod_{k=j+1}^{r-1} \bar{a}_{k}^{q-r-1}-\sum_{i=r+1}^{n} \prod_{k=r+2}^{i} \bar{a}_{k}^{q-r-1}=\Delta_{q-r, q}\left(\bar{a}^{q-r-1}\right)$.

Finally, in view of explicit forms of $\bar{a}_{k}^{s}$ and by the above equalities, we get that differences $\delta_{s}-\delta_{s-1}$, where $s=$ $1,2, \ldots, q-r$, take the following forms:

$$
\begin{array}{ll}
\delta_{1}-\delta_{0} & =\left(a_{q}-a_{q-1}\right) \Delta_{1, q}(a), \\
\delta_{2}-\delta_{1} & =\left(a_{q}-a_{q-2}\right) \Delta_{2, q}(a), \\
\cdots & \cdots \cdots, \\
\delta_{q-r}-\delta_{q-r-1} & =\left(a_{q}-a_{r}\right) \Delta_{q-r, q}(a) .
\end{array}
$$

Summing the above formulae side by side, we obtain formula (12).

Formula (13) can be proved in a similar manner: we start with

$$
\begin{aligned}
\underline{a}^{s}= & \left(\underline{a}_{1}^{s}, \ldots, \underline{a}_{r-1}^{s}, \underline{a}_{r}^{s}, \underline{a}_{r+1}^{s}, \underline{a}_{r+2}^{s},\right. \\
& \left.\ldots, \underline{a}_{q-2}^{s}, \underline{a}_{q-1}^{s}, \underline{a}_{q}^{s}, \underline{a}_{q+1}^{s}, \ldots, \underline{a}_{n}^{s}\right)
\end{aligned}
$$

for $s=0,1,2, \ldots, q-r$ and proceed as previously.

\section{The new necessary condition}

In this section, we present a new necessary condition of schedule optimality for problem $(P)$. This condition will be called the second necessary condition of schedule optimality for problem $(P)$.

\subsection{The first formulation}

The new necessary condition of schedule optimality for problem $(P)$ can be formulated is as follows. 
Theorem 1 Let $a=\left(a_{1}, a_{2}, \ldots, a_{n}\right)$ be an optimal schedule for problem $(P)$. Then, $(i)$ is $V$-shaped, the minimal element in $a$ is $a_{m}$, where $1<m<n$, and (ii) for any $r$ and $q$ such that $1 \leq r<m<q \leq n$ we have

$\Delta_{1, q}(a)=\sum_{j=1}^{q-2} \prod_{k=j}^{q-2} a_{k}-\sum_{i=q+1}^{n} \prod_{k=q+1}^{i} a_{k} \geq 0$

and

$\nabla_{1, r}(a)=\sum_{j=1}^{r-1} \prod_{k=j}^{r-1} a_{k}-\sum_{i=r+2}^{n} \prod_{k=r+2}^{i} a_{k} \leq 0$.

Proof $(i)$ Let $a$ be an optimal schedule for $(P)$. Recall that $n>2$ and $a_{i} \neq a_{j}$ whenever $i \neq j$. The V-shapeness of $a$ follows from Property 3 . To prove that for the minimal element $a_{m}$ in $a$ we have $1<m<n$, we proceed by contradiction.

First, assume that $m=1$. Then, $a_{q+1}-a_{q}>0$ for $q=$ $1,2, \ldots, n-1$. Since $\|C(b)\|_{1}-\|C(a)\|_{1} \geq 0$ for any $b$,

$\sum_{j=0}^{q-1} \prod_{k=j+1}^{q-1} a_{k}-\sum_{i=q+1}^{n} \prod_{k=q+2}^{i} a_{k} \geq 0$

for $q=1,2, \ldots, n-1$. Letting $q=1$ in (16), we have $1 \geq$ $\sum_{i=2}^{n} \prod_{k=3}^{i} a_{k}$. We get a contradiction with the assumption that $a_{k}>1$ and $n>2$.

Now, assume that $m=n$. Then, for $q=n-1$ in (16), we have

$\sum_{j=0}^{n-2} \prod_{k=j+1}^{n-2} a_{k}<1$

since now $a_{q+1}-a_{q}<0$ for $q=1,2, \ldots, n-1$. Again, we get a contradiction with the assumption that $a_{k}>1$ and $n>2$. Hence, $1<m<n$ as required.

(ii) To prove inequality (14), let $m+1 \leq q \leq n$. Moreover, let $r$ in sequence $\bar{a}^{q-r}=\left(a_{1}, \ldots, a_{r-1}, a_{q}, a_{r}, \ldots\right.$, $\left.a_{q-1}, a_{q+1}, \ldots, a_{n}\right)$ be such that $a_{r-1}>a_{q}>a_{r}$. This means that $r$ is $m$-conjugated to $q$. Since $a$ is a $\mathrm{V}$-shaped optimal schedule and since $a_{m}$ is minimal in $a$, we have $a_{r}<a_{q}, a_{r+1}<a_{q}, \ldots, a_{q-1}<a_{q}$ and, as a consequence, $a_{q}-a_{q-k}>0$ for $1 \leq k \leq q-r$. Assume that for some such $q$ there holds the inequality

$\Delta_{1, q}(a)=\sum_{i=1}^{q-2} \prod_{k=i}^{q-2} a_{k}-\sum_{i=q+1}^{n} \prod_{k=q+1}^{i} a_{k}<0$.
Then, by Lemma 1 , for $1 \leq k \leq q-r$ we have

$$
\begin{aligned}
\Delta_{k, q}(a)= & \sum_{i=1}^{q-k-1} \prod_{j=i}^{q-k-1} a_{j}-\sum_{i=q-k+1}^{q-1} \prod_{j=q-k+1}^{i} a_{j} \\
& -\frac{1}{a_{q}} \sum_{j=q+1}^{n} \prod_{j=q-k+1}^{n} a_{j}<0 .
\end{aligned}
$$

Thus, by formula (12), $\left\|C\left(\bar{a}^{q-r}\right)\right\|_{1}-\|C(a)\|_{1}<0$, which contradicts the assumption that $a$ is an optimal schedule. Therefore, for $m+1 \leq q \leq n$, we have $\Delta_{1, q}(a) \geq 0$ and inequality (14) follows.

We prove inequality (15) in a similar way. Let $1 \leq$ $r \leq m-1$. Moreover, let $q$ in sequence $\underline{a}^{q-r}=$ $\left(a_{1}, \ldots, a_{r-1}, a_{r+1}, \ldots, a_{q}, a_{r}, a_{q+1} \ldots, a_{n}\right)$ be such that $a_{q}<a_{r}<a_{q+1}$, i.e. let $q$ be $m$-conjugated to $r$. Since $a$ is a V-shaped optimal schedule and since $a_{m}$ is minimal in $a$, we have $a_{r}>a_{r+1}, a_{r}>a_{r+2}, \ldots, a_{r}>a_{q}$, which implies that $a_{r+k}-a_{r}<0$ for $k=1,2, \ldots, q-r$. Assume that for some such $r$ we have

$\nabla_{1, r}(a)=\sum_{i=1}^{r-1} \prod_{k=i}^{r-1} a_{k}-\sum_{i=r+2}^{n} \prod_{k=r+2}^{i} a_{k}>0$.

Then, by Lemma 1 , for $1 \leq k \leq q-r$ we have

$$
\begin{aligned}
\nabla_{k, r}(a)= & \frac{1}{a_{r}} \sum_{i=1}^{r-1} \prod_{j=i}^{r+k-1} a_{j}+\sum_{i=r+1}^{r+k-1} \prod_{j=i}^{r+k-1} a_{j} \\
& -\sum_{i=r+k+1}^{n} \prod_{j=r+k+1}^{i} a_{j}>0 .
\end{aligned}
$$

Hence, by formula (13), $\left\|C\left(\underline{a}^{q-r}\right)\right\|_{1}-\|C(a)\|_{1}<0$, which contradicts the assumption that $a$ is an optimal schedule. Therefore, we have $\nabla_{1, r}(a) \leq 0$ and inequality (15) follows.

\subsection{The second formulation}

Now, we show that Theorem 1 can be formulated in a slightly different form.

Theorem 2 Let $a=\left(a_{1}, a_{2}, \ldots, a_{n}\right)$ be an optimal schedule for problem $(P)$. Then, $(i)$ a is $V$-shaped, the minimal element in a is $a_{m}$, where $1<m<n$, and (ii) there hold inequalities

$$
\Delta_{1, m+1}(a)=\sum_{j=1}^{m-1} \prod_{k=j}^{m-1} a_{k}-\sum_{i=m+2}^{n} \prod_{k=m+2}^{i} a_{k} \geq 0
$$


and

$\nabla_{1, m-1}(a)=\sum_{j=1}^{m-2} \prod_{k=j}^{m-2} a_{k}-\sum_{i=m+1}^{n} \prod_{k=m+1}^{i} a_{k} \leq 0$.

Proof Let $a=\left(a_{1}, a_{2}, \ldots, a_{n}\right)$ be an optimal schedule for problem $(P)$. The proof of $(i)$ is analogous to that of $(i)$ in Theorem 1.

To prove (ii), notice that if $a$ is $\mathrm{V}$-shaped then, by Theorem 1, there hold inequalities (14) and (15). This, in turn, implies that there hold inequalities (17) and (18), since they are special cases of (14) and (15) for $q=m+1$ and $r=m-1$, respectively.

On the other hand, if inequality (17) holds, then inequality (14) holds as well, since by changing the index $q$ of $\Delta_{1, q}(a)$ from $m+1$ up to $n$ we simultaneously increase the first sum and decrease the second sum what causes that for these indices $\Delta_{1, q}(a) \geq 0$ as required. In a similar way, we show that the decreasing of index $r$ of $\nabla_{1, r}(a)$ in inequality (18) from $m-1$ to 1 implies that $\nabla_{1, r}(a) \leq 0$ as desired in (15), since the first sum decreases and the second sum increases.

Hence, for $m+1 \leq q \leq n$ inequality (14) is equivalent to inequality (17) and for $1 \leq r \leq m-1$ inequality (15) is equivalent to inequality (18).

\section{Comparison of necessary conditions}

In this section, we estimate the power of the first and the second necessary condition of schedule optimality for problem $(P)$, comparing the cardinalities of the sets of schedules which satisfy the respective conditions.

Given an instance $a^{\circ}$ of problem $(P)$, let $V_{\mathrm{I}}\left(a^{\circ}\right)$ and $V_{\mathrm{II}}\left(a^{\circ}\right)$ denote the sets of all $a \in \mathcal{P}\left(a^{\circ}\right)$ that satisfy the first and the second necessary condition, respectively. Definitions of $\mathcal{P}\left(a^{\circ}\right), V_{\mathrm{I}}\left(a^{\circ}\right)$ and $V_{\mathrm{II}}\left(a^{\circ}\right)$ imply that

$V_{\mathrm{II}}\left(a^{\circ}\right) \subseteq V_{\mathrm{I}}\left(a^{\circ}\right) \subset \mathcal{P}\left(a^{\circ}\right)$.

In view of (19), we have

$\left|V_{\mathrm{II}}\left(a^{\circ}\right)\right| \leq\left|V_{\mathrm{I}}\left(a^{\circ}\right)\right|<\left|\mathcal{P}\left(a^{\circ}\right)\right|$,

where $\left|\mathcal{P}\left(a^{\circ}\right)\right|=n$ ! and $\left|V_{\mathrm{I}}\left(a^{\circ}\right)\right|=2^{n-1}$.

Denote

$u=\min \left\{a_{i}^{\circ}: i=1,2, \ldots, n\right\}$

and

$v=\max \left\{a_{i}^{\circ}: i=1,2, \ldots, n\right\}$.
Notice that $1<u<v$. Denote

$d_{n}=n \times \frac{\log u}{\log u+\log v}$

and

$g_{n}=1+n \times \frac{\log v}{\log u+\log v}$.

\subsection{Estimations of the position of $a_{m}$}

In order to calculate the cardinalities of the sets of schedules that satisfy the necessary conditions discussed earlier, we have to estimate the range of possible positions of the minimal element $a_{m}$ in a $\mathrm{V}$-shaped sequence. Before we describe this range, we make two observations.

First, notice that the range of possible values of index $m, 1<m<n$, of the minimal element $a_{m}$ in $a=$ $\left(a_{1}, \ldots, a_{m}, \ldots, a_{n}\right) \in V_{\mathrm{II}}\left(a^{\circ}\right)$ depends on $a$. Second, observe that if the maximal element and the minimal element in $a$ are close to each other, then, roughly speaking, $a_{m}$ is near the middle of $a$. On the other hand, if the difference between the maximal element and the minimal element in $a$ is large enough, then the minimal element $a_{m}$ satisfying the new necessary condition may be located anywhere in $a$.

Theorem 3 Let $a=\left(a_{1}, \ldots, a_{m}, \ldots, a_{n}\right) \in \mathcal{P}\left(a^{\circ}\right)$ be a $V$ shaped schedule for problem $(P)$ such that $a_{m}$ is the minimal element in a. Then, (i) if $1<u<v$ are arbitrary, then $d_{n}<$ $m<g_{n}$; (ii) if $v \rightarrow u$, then $\frac{n}{2} \leq m \leq \frac{n}{2}+1$; (iii) if $v \rightarrow+\infty$, then $1<m<n$.

Proof Let $a=\left(a_{1}, \ldots, a_{m}, \ldots, a_{n}\right) \in \mathcal{P}\left(a^{\circ}\right)$ be a V-shaped schedule for problem $(P)$ and let $a_{m}$ be the minimal element in $a$.

(i) First, we prove that

$\frac{v^{m}-1}{u^{n-m}-1}>1$

and

$\frac{u^{m-1}-1}{v^{n-m+1}-1}<1$.

In order to prove inequality (24), we apply inequality (17), estimating all summands in the first sum from above by $v$ and all summands in the second sum from below by $u$. Next, we compute the sums of two geometric sequences we obtained in that way. In consequence, we have

$\frac{v^{m}-1}{v-1}-\frac{u^{n-m}-1}{u-1} \geq 0$. 
By simplifying the inequality, we obtain that

$\frac{v^{m}-1}{u^{n-m}-1} \geq \frac{v-1}{u-1}>1$

since $1<u<v$ by assumption.

In order to prove inequality (25), we apply inequality (18) and proceed in a similar way. In consequence, we have

$\frac{u^{m-1}-1}{v^{n-m+1}-1} \leq \frac{u-1}{v-1}<1$.

Next, transforming (24) and (25), we inequalities that $m(\log u+\log v)>n \log u$ and $(m-1)(\log u+\log v)<n \log v$. Therefore, $m$ must satisfy the inequalities

$n \frac{\log u}{\log u+\log v}<m<1+n \frac{\log v}{\log u+\log v}$.

Hence, in view of (22) and (23), inequalities in ( $i$ ) follow.

Applying (26) and passing with $v$ to the limits $v \rightarrow u$ and $v \rightarrow+\infty$, we obtain the assertions in (ii) and (iii), respectively.

\subsection{Estimations of $\left|V_{\text {II }}\left(a^{\circ}\right)\right|$}

Theorem 3 implies that the set of possible values of $m$ is contained in the open interval $\left(d_{n}, g_{n}\right)$, where $d_{n}<g_{n}$. The cardinality of the set

$D=\left\{k \in \mathbb{N}: d_{n}<k<g_{n}, 1<k<n\right\}$,

where $\mathbb{N}$ denotes the set of natural numbers, is majorized by $\left\lceil g_{n}\right\rceil-\left\lfloor d_{n}\right\rfloor-1$ (cf. Graham et al. 1989, p. 74).

Let $V_{D}\left(a^{\circ}\right)$ denote the set of all $a=\left(a_{1}, \ldots, a_{m}, \ldots, a_{n}\right)$ $\in \mathcal{P}\left(a^{\circ}\right)$ such that $a$ is $\mathrm{V}$-shaped and $m \in D$. It is clear that

$V_{\mathrm{II}}\left(a^{\circ}\right) \subseteq V_{D}\left(a^{\circ}\right) \subseteq V_{\mathrm{I}}\left(a^{\circ}\right)$

and that

$\left|V_{\mathrm{II}}\left(a^{\circ}\right)\right| \leq\left|V_{D}\left(a^{\circ}\right)\right| \leq\left|V_{\mathrm{I}}\left(a^{\circ}\right)\right|$

Hence, we can estimate the cardinality of the set $V_{\mathrm{II}}\left(a^{\circ}\right)$ by the cardinality of the set $V_{D}\left(a^{\circ}\right)$ which, in view of Property 3 , is estimated by $\left|V_{\mathrm{I}}\left(a^{\circ}\right)\right|=2^{n-1}$.

In order to find $\left|V_{D}\left(a^{\circ}\right)\right|$, we need to know how to generate all V-shaped sequences from a given sequence $a^{\circ}$ with minimal element $a_{m}$, where index $m \in D$. These sequences can be generated as follows. First, we order $a^{\circ}$ non-increasingly. Next, for each $k \in D$, we construct a $\mathrm{V}$-shaped sequence by choosing $k$ elements of $a^{\circ}$ which compose the left branch of the sequence and, in reversed order, $n-k$ remaining elements which compose the right branch of this sequence. However, proceeding in this way, we take into account each V-shaped sequence twice, since the minimal element first is assigned to the left and then to the right branch of the sequence. Hence, since we can choose $k$ elements from $n$ in $\left(\begin{array}{l}n \\ k\end{array}\right)$ ways and since each V-shaped sequence is counted twice, the cardinality of the set $V_{D}\left(a^{\circ}\right)$ equals

$\left|V_{D}\left(a^{\circ}\right)\right|=\frac{1}{2} \sum_{k \in D}\left(\begin{array}{l}n \\ k\end{array}\right)$.

Now, applying Eq. (27), we prove the first main result of the section, which gives a lower and an upper bound of $\left|V_{D}\left(a^{\circ}\right)\right|$ in terms of $u, v$ and $n$.

Theorem 4 Denote $c(n)=\sqrt{\frac{2}{\pi n}} 2^{n-1}\left(1+O\left(\frac{1}{n}\right)\right)$ and let $u$ and $v, 1<u<v$, be defined by (20) and (21), respectively. Then,

$c(n) \leq\left|V_{D}\left(a^{\circ}\right)\right|<\left(2+\frac{\log v-\log u}{\log v+\log u} n\right) \times c(n)$.

Proof Applying definitions (22) and (23) of $d_{n}$ and $g_{n}$, respectively, and knowing that the maximal binomial coefficient either is equal to $\left(\begin{array}{c}n \\ \left\lfloor\frac{n}{2}\right\rfloor\end{array}\right)$ if $n$ is even or it is equal to $\left(\begin{array}{c}n \\ \left\lfloor\frac{n}{2}\right\rfloor\end{array}\right)=\left(\begin{array}{c}n \\ 1+\left\lfloor\frac{n}{2}\right\rfloor\end{array}\right)$ if $n$ is odd, we get that

$$
\begin{aligned}
\left|V_{D}\left(a^{\circ}\right)\right| & =\frac{1}{2} \sum_{k \in D}\left(\begin{array}{l}
n \\
k
\end{array}\right) \leq\left(\left\lceil g_{n}\right\rceil-\left\lfloor d_{n}\right\rfloor-1\right) \times \frac{1}{2}\left(\begin{array}{c}
n \\
\left\lfloor\frac{n}{2}\right\rfloor
\end{array}\right) \\
& <\left(2+\frac{\log v-\log u}{\log v+\log u} n\right) \times \frac{1}{2}\left(\begin{array}{c}
n \\
\left\lfloor\frac{n}{2}\right\rfloor
\end{array}\right) .
\end{aligned}
$$

Notice that $d_{n}<\frac{n}{2}<1+\frac{n}{2}<g_{n}$. This implies that $k=1+\left\lfloor\frac{n}{2}\right\rfloor \in D$. Therefore, there holds inequality

$\left(\begin{array}{c}n \\ \left\lfloor\frac{n}{2}\right\rfloor\end{array}\right) \leq \sum_{k \in D}\left(\begin{array}{l}n \\ k\end{array}\right)$

To show that $c(n) \leq\left|V_{D}\left(a^{\circ}\right)\right|$ and to complete the proof, it is sufficient to apply asymptotic formula $\left(\begin{array}{c}n \\ \left\lfloor\frac{n}{2}\right\rfloor\end{array}\right)=$ $\sqrt{\frac{2}{\pi n}} 2^{n}\left(1+O\left(\frac{1}{n}\right)\right)($ Odlyzko 1995).

From Theorem 4 follow a few corollaries which are important for practice. First, this theorem gives a good lower estimation of $\left|V_{D}\left(a^{\circ}\right)\right|$ and, as a side effect, an upper estimation of $\left|V_{D}\left(a^{\circ}\right)\right|$. The latter, in turn, leads to an upper estimation of $\left|V_{\mathrm{II}}\left(a^{\circ}\right)\right|$. However, if for a given sequence $a$ we have $v \leq u^{\frac{n+1}{n-1}}$, where $u$ and $v$ are defined by (20) and (21), respectively, then

$\left|V_{\mathrm{II}}\left(a^{\circ}\right)\right| \leq 3 \sqrt{\frac{2}{\pi n}}\left(1+O\left(\frac{1}{n}\right)\right) \times\left|V_{\mathrm{I}}\left(a^{\circ}\right)\right|$. 
The latter inequality implies that if $u<v \leq u^{\frac{n+1}{n-1}}$, then the number of sequences satisfying the second necessary condition of optimality for problem $(P)$ is reduced by the factor $O\left(n^{-\frac{1}{2}}\right)$.

Second, if $u$ and $v$ are defined by (20) and (21), respectively, and $v \searrow u$, then $d_{n} \nearrow \frac{n}{2} \leq m \leq 1+\frac{n}{2} \swarrow g_{n}$ simultaneously. This means that if $v$ is close to $u$, then we can expect that the cardinality of the set $D$ becomes smaller, so the set $V_{D}\left(a^{\circ}\right)$, and hence the set $V_{\mathrm{II}}\left(a^{\circ}\right)$, becomes smaller as well. In other words, if elements of a given sequence $a^{\circ}$ are close each to other, then the set of all $\mathrm{V}$-shaped sequences satisfying the second necessary condition is smaller, which makes the verification of this condition a more simple task.

Third, if for a given sequence $a^{\circ}$ we have $u<<v$, then the set $D$, and hence the set $V_{D}\left(a^{\circ}\right)$, becomes larger and verification of the second necessary condition can be a more expensive task. More formally, if $1<u<v$ and $v \rightarrow+\infty$, then the set $D$ approaches the set $\{2, \ldots, n-1\}$ and, in consequence, $\left|V_{\mathrm{II}}\left(a^{\circ}\right)\right|$ may be large, since the set $V_{D}\left(a^{\circ}\right)$ can be larger.

Now, applying an asymptotic formula for partial binomial sums (cf. Graham et al. 1989), we prove the second main result of this section, which gives another estimation of $\left|V_{\mathrm{II}}\left(a^{\circ}\right)\right|$ from above.

Let

$H(t)=t \log _{2} \frac{1}{t}+(1-t) \log _{2}\left(\frac{1}{1-t}\right)$

denote the binary entropy function and let

$\bar{H}(t)=1-H(t)$,

where $t \in[0,1]$. Notice that $0 \leq H(t) \leq 1$ for $0 \leq t \leq 1$ and that the maximum of the function equals to $H\left(\frac{1}{2}\right)=1$.

Lemma 3 Given any constants $\lambda$ and $\mu$ such that $0<\lambda<\frac{1}{2}$ and $\frac{1}{2}<\mu<1$, we have

$$
\sum_{0 \leq k \leq \lambda n}\left(\begin{array}{l}
n \\
k
\end{array}\right)=2^{n H(\lambda)-\frac{1}{2} \log _{2} n+O(1)}
$$

and

$$
\sum_{\mu n \leq k \leq n}\left(\begin{array}{l}
n \\
k
\end{array}\right)=2^{n H(1-\mu)-\frac{1}{2} \log _{2} n+O(1)} .
$$

Proof Formula (28) is given by Graham et al (1989, p. 492). In order to prove formula (29), we apply the following symmetric formula for partial sum of binomials:

$$
\sum_{\mu n \leq k \leq n}\left(\begin{array}{l}
n \\
k
\end{array}\right)=\sum_{0 \leq l \leq(1-\mu) n}\left(\begin{array}{l}
n \\
l
\end{array}\right),
$$

where $\frac{1}{2}<\mu<1$. Indeed, observe that $\left(\begin{array}{l}n \\ k\end{array}\right)=\left(\begin{array}{c}n \\ n-k\end{array}\right)$. Applying substitution $l=n-k$, we get the above formula. To end the proof it is sufficient to apply formula (28) with $\lambda=1-\mu$, since then we have $0<\lambda<\frac{1}{2}$.

Applying Lemma 3, we can prove the another upper bound of $\left|V_{\mathrm{II}}\left(a^{\circ}\right)\right|$. Let $n$ be fixed but arbitrary natural number and define

$\alpha=\frac{\log u}{\log u+\log v}$ and $\beta=\frac{\log v}{\log u+\log v}$.

Clearly, $\alpha+\beta=1,0<\alpha<\frac{1}{2}$ and $\frac{1}{2}<\beta<1$.

Theorem 5 Let $u$ and $v$ be defined by formulae (20) and (21), respectively, and let

$\Lambda_{n}(u, v)=1-2^{-n \bar{H}\left(\frac{\log u}{\log u+\log v}\right)-\frac{1}{2} \log _{2} n+O(1)}$.

Then, for any $n>2$, we have

$\left|V_{\mathrm{II}}\left(a^{\circ}\right)\right| \leq \Lambda_{n}(u, v) \times\left|V_{\mathrm{I}}\left(a^{\circ}\right)\right|$

and

$0<\Lambda_{n}(u, v)<1$,

whenever $1<u<v$.

Proof First, notice that for $d_{n}$ defined by formula (22) we have $\alpha n=d_{n}$, where $0<\alpha<\frac{1}{2}$. Similarly, for $g_{n}$ defined by formula (23) we have $g_{n}=1+\beta n$, where $\frac{1}{2}<\beta<1$.

Second, we have

$$
\begin{aligned}
2^{n} & =\sum_{k=0}^{n}\left(\begin{array}{l}
n \\
k
\end{array}\right) \\
& =\sum_{0 \leq k \leq d_{n}}\left(\begin{array}{l}
n \\
k
\end{array}\right)+\sum_{d_{n}<k<g_{n}}\left(\begin{array}{l}
n \\
k
\end{array}\right)+\sum_{g_{n} \leq k \leq n}\left(\begin{array}{l}
n \\
k
\end{array}\right) \\
& =\sum_{0 \leq k \leq \alpha n}\left(\begin{array}{l}
n \\
k
\end{array}\right)+\sum_{d_{n}<k<g_{n}}\left(\begin{array}{l}
n \\
k
\end{array}\right)+\sum_{1+\beta n \leq k \leq n}\left(\begin{array}{l}
n \\
k
\end{array}\right) .
\end{aligned}
$$

Since $\left(\begin{array}{l}n \\ k\end{array}\right)=\frac{n}{k}\left(\begin{array}{c}n-1 \\ k-1\end{array}\right)$ and $\frac{n}{k} \geq 1$ we get, after substitution $l=k-1$, that

$\sum_{1+\beta n \leq k \leq n}\left(\begin{array}{l}n \\ k\end{array}\right) \geq \sum_{\beta n \leq l \leq n-1}\left(\begin{array}{c}n-1 \\ l\end{array}\right)$.

Applying Lemma 3, with $\lambda=\alpha$ and $\mu=\beta$, we obtain 


$$
\begin{aligned}
2^{n} \geq & \sum_{d_{n}<k<g_{n}}\left(\begin{array}{l}
n \\
k
\end{array}\right)+2^{n H(\alpha)-\frac{1}{2} \log _{2} n+O(1)} \\
& +2^{(n-1) H(1-\beta)-\frac{1}{2} \log _{2}(n-1)+O(1)} \\
\geq & \sum_{d_{n}<k<g_{n}}\left(\begin{array}{l}
n \\
k
\end{array}\right)+2^{n H(\alpha)-\frac{1}{2} \log _{2} n+O(1)},
\end{aligned}
$$

where in order to obtain the second inequality we have applied the equality $1-\beta=\alpha$ and inequality $\frac{1}{2} \log \left(\frac{n}{n-1}\right) \geq 0$, while the last term has entered into the second one in view of $0 \leq H(\alpha) \leq 1$ together with $O(1)+$ const $=O(1)$.

Therefore, for any $n>2$ we have

$$
\begin{aligned}
\left|V_{D}\left(a^{\circ}\right)\right| & =\frac{1}{2} \sum_{d_{n}<k<g_{n}}\left(\begin{array}{l}
n \\
k
\end{array}\right) \leq 2^{n-1}\left(1-2^{-n \bar{H}(\alpha)-\frac{1}{2} \log _{2} n+O(1)}\right) \\
& =\Lambda_{n}(u, v) \times 2^{n-1} .
\end{aligned}
$$

Hence, since $\left|V_{\mathrm{I}}\left(a^{\circ}\right)\right|=2^{n-1}$ and $\left|V_{\mathrm{II}}\left(a^{\circ}\right)\right| \leq\left|V_{D}\left(a^{\circ}\right)\right|$, inequality (30) follows.

To complete the proof, it is sufficient to note that $\Lambda_{n}(u, v)$ is a ratio greater than 0 and less than 1 , whenever $1<u<v$.

From Theorem 5 follow a few corollaries. Let $a^{\circ}$ be a given sequence and let $1<u<v$. Then, first, $\left|V_{\mathrm{II}}\left(a^{\circ}\right)\right|<\left|V_{\mathrm{I}}\left(a^{\circ}\right)\right|$ since $0<\Lambda_{n}(u, v)<1$.

Second, in view of inequality (30), $\left|V_{\mathrm{II}}\left(a^{\circ}\right)\right|$ is controlled from above by $\Lambda_{n}(u, v)$.

Third, if $v$ is close to $u$, then $V_{\mathrm{II}}\left(a^{\circ}\right)$ is essentially smaller than $V_{\mathrm{I}}\left(a^{\circ}\right)$. Indeed, if $v \searrow u$, then $\bar{H}\left(\frac{\log u}{\log u+\log v}\right)$ approaches zero and then

$\Lambda_{n}(u, v) \nearrow 1-\frac{1}{\sqrt{n}} \times 2^{O(1)}$,

which implies that $V_{\mathrm{II}}\left(a^{\circ}\right)$ is essentially smaller than $V_{\mathrm{I}}\left(a^{\circ}\right)$.

Finally, if $v$ is large, then it may happen that the set $V_{\mathrm{II}}\left(a^{\circ}\right)$ approaches the set $V_{\mathrm{I}}\left(a^{\circ}\right)$. Indeed, if $v \nearrow+\infty$, then

$\Lambda_{n}(u, v) \searrow 1-\frac{2^{-n}}{\sqrt{n}} \times 2^{O(1)}$,

since $\bar{H}\left(\frac{\log u}{\log u+\log v}\right) \nearrow 1$. Hence, $V_{\mathrm{II}}\left(a^{\circ}\right)$ can be 'close' to $V_{\mathrm{I}}\left(a^{\circ}\right)$.

\section{Numerical examples}

In this section, we illustrate the results of Sect. 5 with a few examples.

The first example shows how large is set $V_{\mathrm{II}}\left(a^{\circ}\right)$ compared to set $V_{\mathrm{I}}\left(a^{\circ}\right)$, when $a^{\circ}$ is a regular instance composed of consecutive positive integer numbers. Let
$r_{\mathrm{II}}\left(a^{\circ}\right)=100 \times \frac{\left|V_{\mathrm{II}}\left(a^{\circ}\right)\right|}{\left|V_{\mathrm{I}}\left(a^{\circ}\right)\right|}$

denote the ratio of the size of set $V_{\mathrm{II}}\left(a^{\circ}\right)$ and the size of set $V_{\mathrm{I}}\left(a^{\circ}\right)$.

Example 1 Let $n=9$ and $a=(10,9,8,7,6,5,4,3,2)$. Then, by Property 1 , we can limit further considerations only to V-shaped sequences starting with 10 . Hence, we can assume that $a^{\circ}=(9,8,7,6,5,4,3,2)$. Then, we have $\left|\mathcal{P}\left(a^{\circ}\right)\right|=40,320,\left|V_{\mathrm{I}}\left(a^{\circ}\right)\right|=128$ and $\left|V_{\mathrm{II}}\left(a^{\circ}\right)\right|=20$. Hence, $r_{\mathrm{II}}\left(a^{\circ}\right)=15.62$.

Optimal V-shaped sequences are in the form of

$a^{\star}=(10,9,6,5,2,3,4,7,8)$

and

$\bar{a}^{\star}=(10,8,7,4,3,2,5,6,9)$,

both with the total completion time equal to 473,945 .

Figure 1 depicts, along the horizontal axis, all $128=2^{7}$ V-shaped schedules from $V_{\mathrm{I}}\left(a^{\circ}\right) \subset \mathcal{P}\left(a^{\circ}\right)$. On the vertical axis there are given total completion times. Bold dots correspond to sequences satisfying the second necessary condition. Notice that the graph presented in Fig. 1 is symmetric in view of Property 2.

Example 1 shows that set $V_{\mathrm{II}}\left(a^{\circ}\right)$ for medium size regular instances is a small subset of set $V_{\mathrm{I}}\left(a^{\circ}\right)$. The next two examples show that a similar claim may be formulated also for larger regular instances.

Example 2 Let $n=15$ and $a=(16,15, \ldots, 2)$. Then, $a^{\circ}=$ $(15,13, \ldots, 2),\left|\mathcal{P}\left(a^{\circ}\right)\right|=8.717829 \times 10^{10},\left|V_{\mathrm{I}}\left(a^{\circ}\right)\right|=$ 8,192 and $\left|V_{\mathrm{II}}\left(a^{\circ}\right)\right|=1,066$. Hence, $r_{\mathrm{II}}\left(a^{\circ}\right)=13.01$, i.e. the size of set $V_{\mathrm{II}}\left(a^{\circ}\right)$ equals about $13 \%$ of the size of set $V_{\mathrm{I}}\left(a^{\circ}\right)$.

In this case, optimal V-shaped sequences are in the form of

$a^{\star}=(16,15,12,11,8,7,4,3,2,5,6,9,10,13,14)$

and

$\bar{a}^{\star}=(16,14,13,10,9,6,5,2,3,4,7,8,11,12,15)$,

both with the total completion time equal to $1,511,666,077$, 882.

Example 3 If we increase the size of $a$ to $n=16$, then $a=(17,16, \ldots, 2), a^{\circ}=(16,15, \ldots, 2),\left|\mathcal{P}\left(a^{\circ}\right)\right|=$ $1.307674 \times 10^{12},\left|V_{\mathrm{I}}\left(a^{\circ}\right)\right|=16,384$ and $\left|V_{\mathrm{II}}\left(a^{\circ}\right)\right|=2,060$. This implies that $r_{\mathrm{II}}\left(a^{\circ}\right)=12.57$, i.e. the size of set $V_{\mathrm{II}}\left(a^{\circ}\right)$ equals only about $12.5 \%$ of the size of set $V_{\mathrm{I}}\left(a^{\circ}\right)$. 


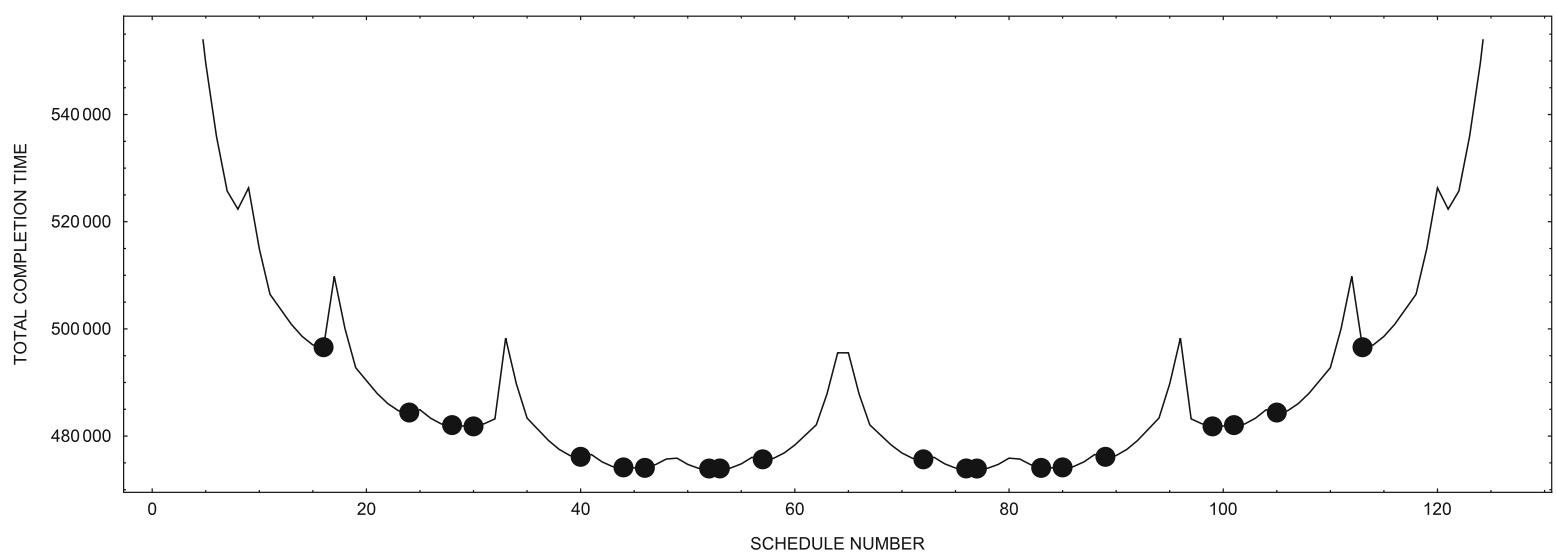

Fig. $1 V_{\mathrm{I}}\left(a^{\circ}\right)$ versus $V_{\mathrm{II}}\left(a^{\circ}\right)$

Optimal V-shaped sequences are in the form of

$a^{\star}=(17,16,13,12,9,8,5,4,2,3,6,7,10,11,14,15)$

and

$\bar{a}^{\star}=(17,15,14,11,10,7,6,3,2,4,5,8,9,12,13,16)$,

both with the total completion time equal to $23,947,081,624$, 255.

The next two examples concern random instances with components from set $\{1,2, \ldots, 99\}$. Though sets $V_{\mathrm{II}}\left(a^{\circ}\right)$ in the examples are slightly larger compared to those for regular sequences, ratio $r_{\mathrm{II}}\left(a^{\circ}\right)$ is still small.

Example 4 Let $n=15$ and $a=(97,92,87,82,65,45,37,32$, $28,27,26,22,19,14,3)$. Then, $a^{\circ}=(92,87,82,65,45,37$, $32,28,27,26,22,19,14,3),\left|V_{\mathrm{I}}\left(a^{\circ}\right)\right|=8,192$ and $\left|V_{\mathrm{II}}\left(a^{\circ}\right)\right|$ $=1,214$, i.e. ratio $r_{\mathrm{II}}\left(a^{\circ}\right)=14.82$.

In this case, optimal V-shaped sequences are in the form of

$a^{\star}=(97,92,65,45,28,27,19,14,3,22,26,32,37,82,87)$

and

$\bar{a}^{\star}=(97,87,82,37,32,26,22,3,14,19,27,28,45,65,92)$

both with the total completion time equal to $8.022563 \times 10^{20}$.

Example 5 Let $n=16$ and $a=(95,90,82,77,75,72,70$, $68,62,60,56,41,29,26,20,12)$. Then, $a^{\circ}=(90,82,77$, $75,72,70,68,62,60,56,41,29,26,20,12), \quad\left|V_{\mathrm{I}}\left(a^{\circ}\right)\right|=$ 16,384 , and $\left|V_{\mathrm{II}}\left(a^{\circ}\right)\right|=3,380$, i.e. ratio $r_{\mathrm{II}}\left(a^{\circ}\right)=20.63$.

Optimal V-shaped sequences are in the form of

$a^{\star}=(95,90,75,70,62,60,29,26,12,20,41,56,68,72,77,82)$ and

$\bar{a}^{\star}=(95,82,77,72,68,56,41,20,12,26,29,60,62,70,75,90)$

both with the total completion time equal to $2.311223 \times 10^{25}$.

We complete this section with results of a numerical experiment, in which 220 random instances of size $n=$ $6,7, \ldots, 16$ were tested. The experiment was conducted using a code implemented in GNU Octave version 4.4.1, running under 64-bit Windows 10 Pro operating system on a computer with Intel Core $1.80 \mathrm{GHz}$ CPU and $16 \mathrm{~GB}$ RAM.

Components of all instances were randomly generated from set $\{1,2, \ldots, 99\}$ without repetition, i.e. in each generated instance no component could be repeated more than once. For a given $n$, the average ratio $r_{\mathrm{II}}\left(a^{\circ}\right)$ was computed for all instances $a^{\circ}$ generated in $k=5$ or $k=15$ independent runs.

The average computation time for a single instance grown geometrically with a factor about 2 , varying between ca. $275 \mathrm{~ms}$ for $n=6$ and ca. $283 \mathrm{~s}$ for $n=16$.

Table 1 presents results for $k=5$ and $k=15$ runs, symbol $r_{\text {II }}(k)$ denotes the average ratio $r_{\text {II }}\left(a^{\circ}\right)$ for all instances of a given size generated in $k$ runs.

Examples $1-5$ and Table 1 show that for many instances of problem $(P)$ the number of schedules satisfying the new condition is significantly smaller compared to the previous necessary condition. Therefore, the claim that the new necessary condition is stronger than the previous one seem to be fully justified.

\section{Conclusions}

In this paper, we have considered the problem of minimizing the total completion time for a set of independent and linearly deteriorating jobs scheduled on a single machine. First, we 
Table 1 Average ratios $r_{\mathrm{II}}\left(a^{\circ}\right)$

\begin{tabular}{lll}
\hline$n$ & $r_{\text {II }}(5)$ & $r_{\text {II }}(15)$ \\
\hline 6 & 37.50 & 36.67 \\
7 & 20.00 & 18.75 \\
8 & 31.25 & 30.21 \\
9 & 17.19 & 16.56 \\
10 & 26.25 & 26.56 \\
11 & 14.77 & 15.34 \\
12 & 18.44 & 12.51 \\
13 & 13.75 & 13.95 \\
14 & 20.96 & 18.30 \\
15 & 13.64 & 13.10 \\
16 & 18.84 & 13.63 \\
\hline
\end{tabular}

proved some results concerning the impact of cyclic shifts of job sequences on the value of the criterion function. Next, based on these results, we proved a new, stronger, necessary condition of schedule optimality for the considered problem, reducing the number of schedules that we must check while looking for an optimal schedule, compared to the previous necessary condition for this problem. Finally, we estimated the size of this reduction, giving new bounds on the cardinality of the set of all possible optimal schedules for the problem.

Our results show that the new necessary condition is stronger than the previous necessary condition, since for many instances of problem $(P)$ the number of schedules satisfying the new condition is significantly smaller compared to the previous one. Therefore, the set of possible optimal solutions of the problem can be narrowed. Hence, the new necessary condition can be used to discard non-promising solutions in exact and sub-optimal algorithms.

Future research may concern the status of time complexity of problem $(P)$ or algorithms that effectively generate schedules satisfying the new necessary condition of optimality for the problem. Another interesting topic for future research is development of sub-optimal algorithms for $(P)$, which start with an initial $\mathrm{V}$-shaped schedule $a$ and applying alternately the cyclic shifts $a(q \leftarrow r)$ or $a(q \rightarrow r)$ generate new $\mathrm{V}$-shaped schedules with lower and lower total completion times.

Open Access This article is licensed under a Creative Commons Attribution 4.0 International License, which permits use, sharing, adaptation, distribution and reproduction in any medium or format, as long as you give appropriate credit to the original author(s) and the source, provide a link to the Creative Commons licence, and indicate if changes were made. The images or other third party material in this article are included in the article's Creative Commons licence, unless indicated otherwise in a credit line to the material. If material is not included in the article's Creative Commons licence and your intended use is not permitted by statutory regulation or exceeds the permitted use, you will need to obtain permission directly from the copy- right holder. To view a copy of this licence, visit http://creativecomm ons.org/licenses/by/4.0/.

\section{References}

Agnetis, A., Billaut, J.-C., Gawiejnowicz, S., Pacciarelli, D., \& Soukhal, A. (2014). Multiagent scheduling: Models and algorithms. Berlin: Springer.

Cheng, T.-C. E., Ding, Q., \& Lin, B. M.-T. (2004). A concise survey of scheduling with time-dependent processing times. European Journal of Operational Research, 152(2004), 1-13.

Gawiejnowicz, S. (2008). Time-dependent scheduling. Berlin: Springer.

Gawiejnowicz, S. (2020a). A review of four decades of time-dependent scheduling: Main results, new topics, and open problems. Scheduling, 23, 3-47.

Gawiejnowicz, S. (2020b). Models and algorithms of time-dependent scheduling. Berlin: Springer.

Gawiejnowicz, S., \& Kurc, W. (2015). Structural properties of timedependent scheduling problems with the $l_{p}$ norm objective. Omega International Journal of Management Science, 57, 196-202.

Gawiejnowicz, S., Kurc, W., \& Pankowska, L. (2006a). Analysis of a time-dependent scheduling problem by signatures of deterioration rate sequences. Discrete Applied Mathematics, 154, 2150-2166.

Gawiejnowicz, S., Kurc, W., \& Pankowska, L. (2006b). Pareto and scalar bicriterion optimization in scheduling deteriorating jobs. Computers \& Operations Research, 33, 746-767.

Graham, R. L., Knuth, D. E., \& Patashnik, O. (1989). Concrete mathematics (2nd ed.). Reading, MA: Addison-Wesley.

Kubale, M., \& Ocetkiewicz, K. M. (2009). A new optimal algorithm for a time-dependent scheduling problem. Control and Cybernetics, $38,713-721$.

Mosheiov, G. (1991). V-shaped policies for scheduling deteriorating jobs. Operational Research, 39, 979-991.

Mosheiov, G. (1994). Scheduling jobs under simple linear deterioration. Computers \& Operations Research, 21, 653-659.

Ocetkiewicz, K. (2010). A FPTAS for minimizing total completion time in a single machine time-dependent scheduling problem. European Journal of Operational Research, 203, 316-320.

Ocetkiewicz, K. (2013). Partial dominated schedules and minimizing the total completion time of deteriorating jobs. Optimization, 62, $1341-1356$.

Odlyzko, A. M. (1995). Asymptotic enumeration methods. In R. L. Graham, M. Groetschel, \& L. Lovasz (Eds.), Handbook of combinatorics (Vol. 2, pp. 1063-1229). Amsterdam: Elsevier.

Strusevich, V. A., \& Rustogi, K. (2017). Scheduling with times-changing effects and rate-modifying activities. Berlin: Springer.

Publisher's Note Springer Nature remains neutral with regard to jurisdictional claims in published maps and institutional affiliations. 